\title{
Lymphangioma of the Foot: A case report
}

\author{
by Steven F. Boc, DPM,FACFAS,FACFAOM ${ }^{1 \rrbracket}$, Panagiotis Panagakos, DPM $^{2}$, Soorena Sadri, DPM ${ }^{3}$,
}

The Foot and Ankle Online Journal 4 (2): 1

Vascular Malformations (VM) are localized or diffuse errors of embryonic development that may affect any segment of the vascular tree, including arterial, venous, capillary and lymphatic vessels. Lymphangiomas are rare vascular lymphatic malformations (LM). The rate of occurrence in the foot and ankle regions has been scarcely reported. Lymphangiomas are slow flow vascular malformations and are histological classified into capillary, cavernous and cystic. Treatment options for lymphangiomas include schlerotherapy, the use of angiogenesis inhibitors; however complete surgical resection is the recommended treatment.

Key words: Lymphangioma, tumor, lymphatic malformations, vascular malformation, soft tissue mass, hemangioma, foot, vascular anomaly.

Published: February, 2011

This is an Open Access article distributed under the terms of the Creative Commons Attribution License. It permits unrestricted use, distribution, and reproduction in any medium, provided the original work is properly cited. (The Foot and Ankle Online Journal (www.faoj.org)

L ymphangiomas are rare soft tissue vascular malformations that can appear on the body. ${ }^{1,2}$ Lymphangiomas of the foot and ankle is a rare occurrence. ${ }^{3}$ Lymphangiomas are equally distributed among genders and races. In the literature, their incidence in children is estimated to amount to $6 \%$ of all benign tumors. In about half of the patients the disease is already obvious at the time of birth. The tumor commonly appears as a ballotable mass and the overlying skin is usually normal, but may have a bluish hue. Lymphangiomas of the extremity can cause diffuse swelling or localized swelling or gigantism with soft tissue and skeletal overgrowth. They are commonly misdiagnosed as Hemangiomas. ${ }^{1,4,5,6}$

Address correspondence to: Steven F. Boc, DPM, FACFAS, FACFAOM, 235 North Broad Street, Philadelphia, PA 19107.

${ }^{1}$ Director of Podiatric Medicine and Surgery Residency Program, Hahnemann University Hospital/Associate Professor Department of Surgery Drexel College of Medicine.

${ }^{2}$ PGY3 Podiatric Surgery and Medicine Resident, Hahnemann University Hospital/Drexel College of Medicine.

${ }^{3}$ PGY2 Podiatric Surgery and Medicine Resident, Hahnemann University

Hospital/Drexel College of Medicine.
Vascular malformations (VM) are localized or diffuse errors of embryonic development that may affect any segment of the vascular tree, including arterial, venous, capillary and lymphatic vessels. During Embryogenesis the development of the vascular system occurs by two separate but related processes; vasculogenesis and angiogenesis. Development of the lymphatic system begins in the sixth to seventh week of gestation, approximately four weeks after the onset of vasculogenesis. Existing veins give rise to lymph sacs which then bud lymphatic capillaries in a centrifugal manner. There is an in situ differentiation of lymphangioblasts from mesenchymal cells into lymphatic endothelial cells with subsequent recruitment of these cells into developing lymphatic vessels. 


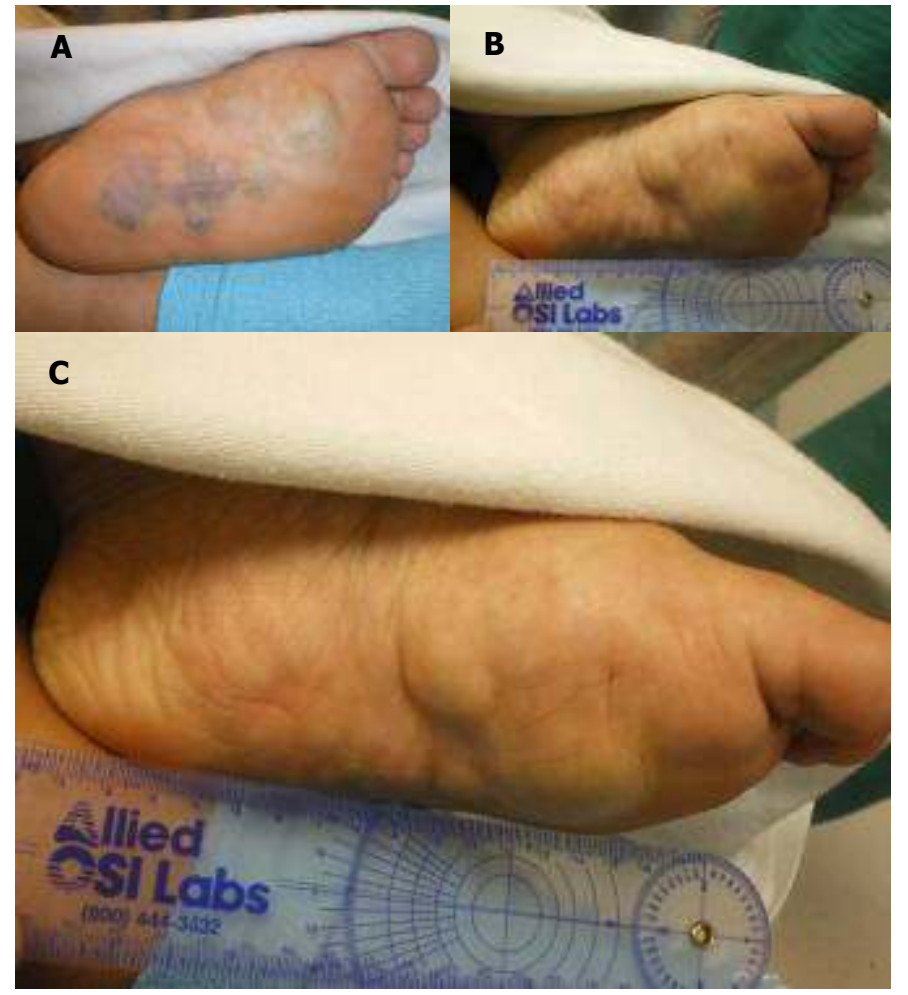

Figure 1A, 1B and 1C Initial presentation of lymphangioma on plantar aspect of foot.(A), A more oblique view of the lesion. (B). A more lateral view of the lesions. (C)

Vasculogenesis, angiogenesis and lymphangiogenesis are subject to precise regulation of growth factors, intercellular and extracellular matrix signaling molecules including vascular endothelial growth factor (VEGF) and the VEGF receptor families, angiopoietins and the Tie- 2 receptor, transforming growth factor- $\beta$ and its receptor, PDGF-B and its receptor, the Notch and Jagged families of membrane associated molecules and the integrin family of cell surface receptors. The integrins which mediate interactions within the extracellular matrix are important in the formation of the vascular and lymphatic systems. Mutations or deletions in specific integrin subtypes can lead to abnormal lymphatic development. ${ }^{4,5}$ In 2007 Skandalakis, et al., described the formation of the lymphatic system which they clearly stated is an enigma. They report the formation of a lymphangiomas as a very rare phenomenon. ${ }^{2}$
The greatest impediment for the diagnosis and treatment of vascular anomalies has been the confusing terminology. ${ }^{5}$ A biologic classification system introduced in 1982 based on studies correlating physical findings, natural history and cellular features has clarified most of the terminologic disorder. There are two major types of vascular anomalies: tumors and malformations. Vascular tumors are endothelial neoplasms characterized by increased cellular proliferation. Hemangioma is the most common and is almost exclusive to infants. Vascular malformations are the result of abnormal development of vascular elements during embryogenesis and fetal life. These may be single forms (capillary, arterial, lymphatic or venous) or a combination. Vascular malformations do not generally demonstrate increased endothelial turnover. They are designated according to the predominant channel type as capillary malformations, lymphatic malformations (LMs), venous malformations (VMs), arteriovenous malformations, and complex forms such as capillary-lymphatico-venous malformations. Malformations with an arterial component are fastflow while the remainders are slow-flow. ${ }^{4,5}$

\section{Case Report}

A 25 year-old female presented to our office in April of 2009 with complaints of multiple discolored and enlarged painful masses on the plantar aspect of her left foot. (Figs 1A, 1B, and 1C) The masses have been present since early childhood but recently have caused her pain while ambulating. Radiographs were obtained upon initial presentation but were unremarkable. Aspiration of the masses was attempted in the office. The aspirate contained dark red fluid which was sent for culture, which came back negative for any infective process. It was decided at this point to send the patient for a series of diagnostic exams. Bilateral lower extremity venous ultrasound resulted in no evidence of a deep vein thrombosis (DVT). Bilateral ankle brachial indexes were unremarkable as well. 


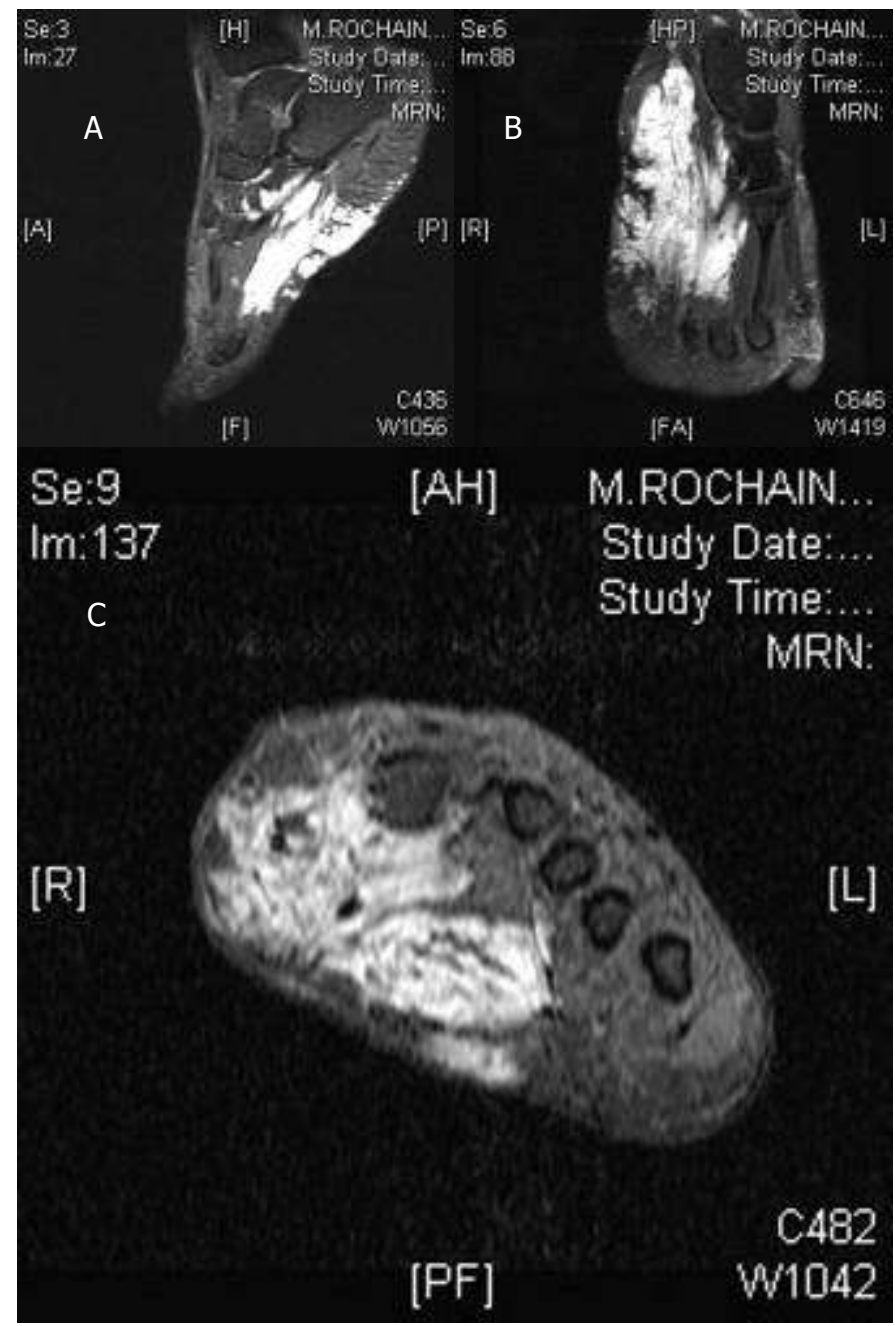

Figure 2A, 2B and 2C MRI of lymphangioma; sagittal view $(A)$, Axial view (B) and Coronal view. (C)

Magnetic resonance imaging (MRI) was performed for the left foot which showed an enhancing soft tissue mass involving the quadrates plantae, flexor digitorum brevis and flexor hallucis muscle bellies consistent with a hemangioma. (Figs. 2A, 2B and 2C) Due to the extensive nature of the soft tissue masses and severe pain it was determined that surgical excision be performed. The patient received a popliteal nerve block pre operatively for pain control. One plantar incision was used to access the most painful soft tissue masses. It was noted that once the ankle tourniquet was inflated the masses decreased in size.

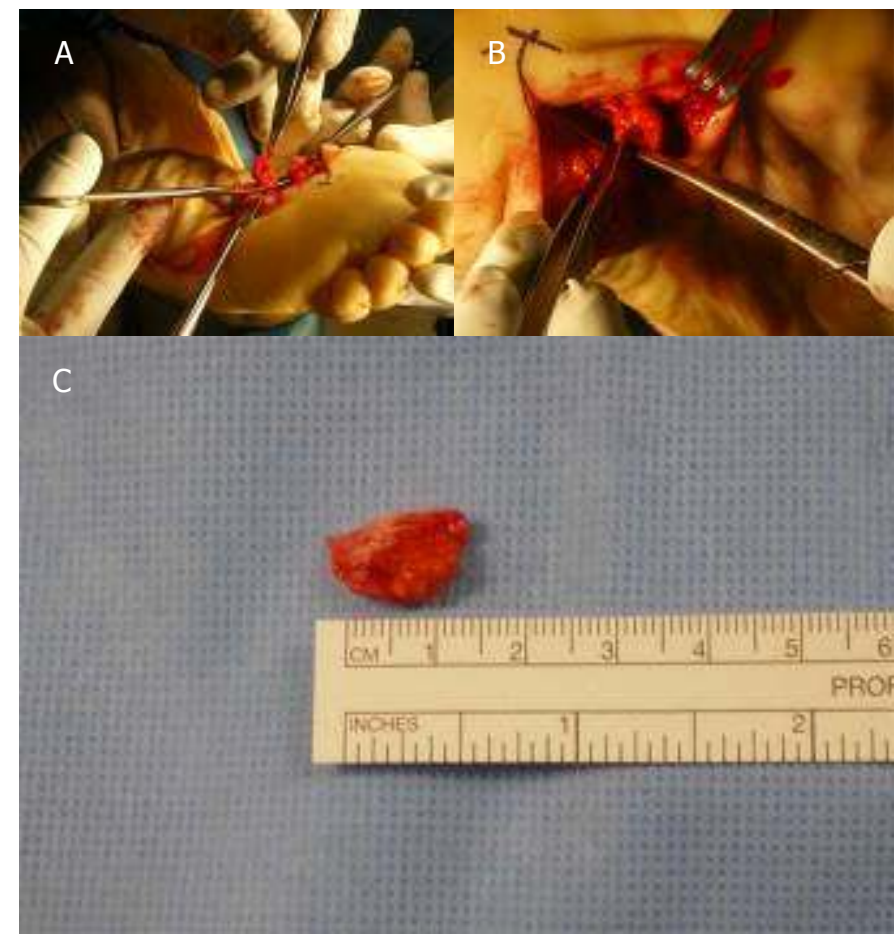

Figure 3A, 3B and $3 C$ Intra operative removal of the lymphangioma ( $A$ and $B$ ). Removed lymphangioma on the back table. (C)

The masses were excised and sent to pathology for evaluation. (Figs. 3A, 3B and 3C) The final report by the pathologist was dense fibrocollagenous tissue, nerve bundles and few dilated endothelial lined spaces and enlarged lymphatic sacs suggestive of a lymphangioma. (Figs. 4A and 4B)

The patient returned the next day to the emergency department in excruciating pain and unable to bear weight on the left foot. There were no signs of infection present at this time (Fig. 5A). Patient was admitted for pain management and was discharged 4 days later when her pain was controlled by simple oral analgesics. Patient presented to the office three weeks after excision with a dehiscence of the surgical site. She was admitted at this point and an incision and drainage with primary closure was performed during this hospital stay (Fig. 5A and 5B). She returned approximately 2 weeks after her second admission to our office. 


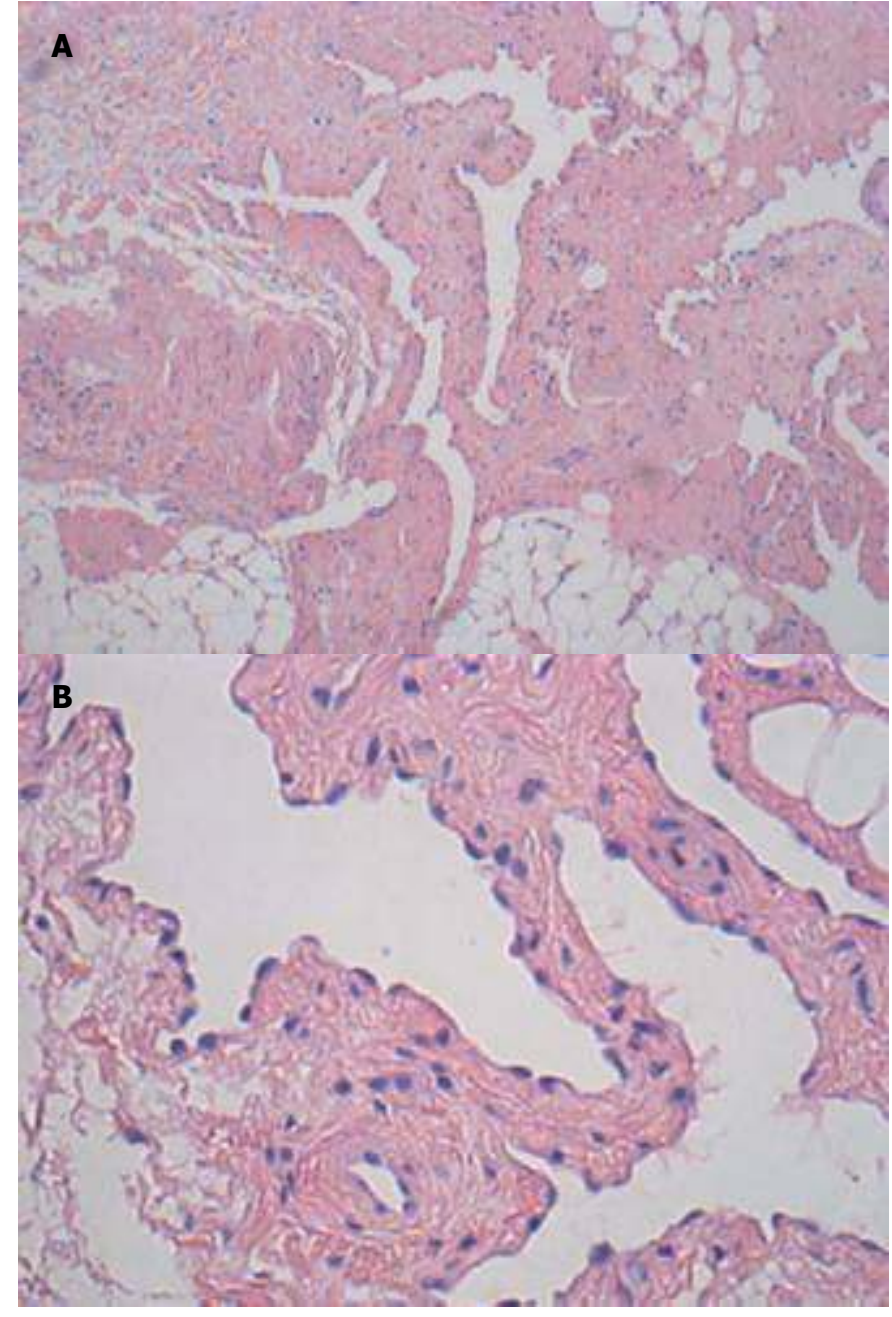

Figure 4A and 4B 10x hematoxylin \& eosin slide, shows the presence of many dilated vascular channels lined by flattened endothelial cells and containing lymphatic fluid. (A) The 40x hematoxylin \& eosin slide, shows the presence of many dilated vascular channels lined by flattened endothelial cells and containing lymphatic fluid.

At this point there were no signs of infection and the sutures were removed. (Figs. 6A and 6B) The surgical site appeared healed and no evidence of recurrence of the lymphangioma was apparent at this point. The patient was lost to follow up after this office visit.

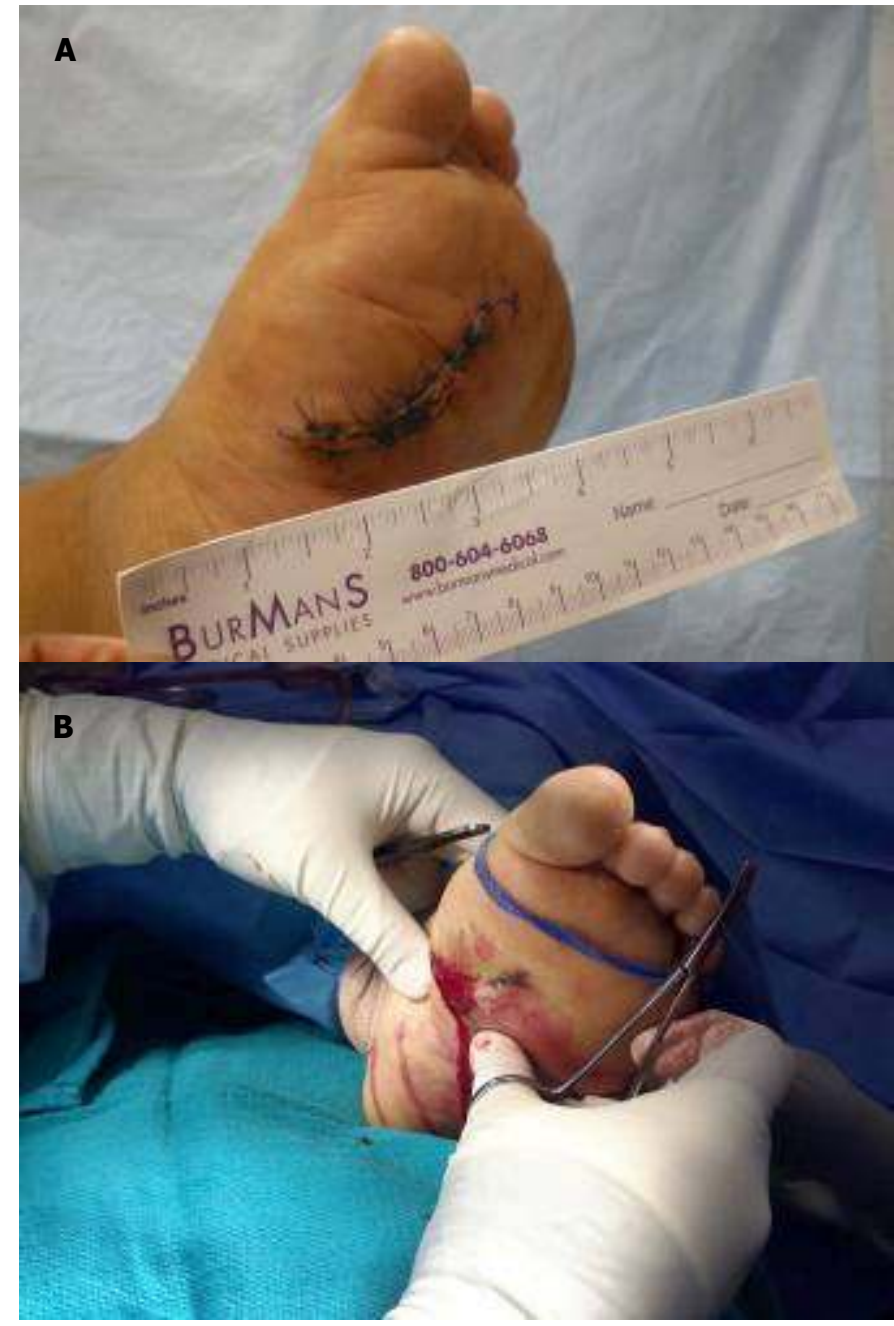

Figure 5A and 5B One day excision of the lymphangioma. (A) The patient returned to the operating suite for operative incision and drainage of additional serous fluid with primary closure.(B)

\section{Discussion and Conclusion}

Lymphangiomas are usually noted at birth or within 2 years of life. ${ }^{1} \quad$ Prenatal ultrasonography can detect relatively large lesions as early as the second trimester, although lymphangiomas are frequently misdiagnosed as other pathologic entities. Lymphangiomas most commonly occur in the cervicofacial region, axilla/chest, mediastinum, retroperitoneum, buttock and perineum. 


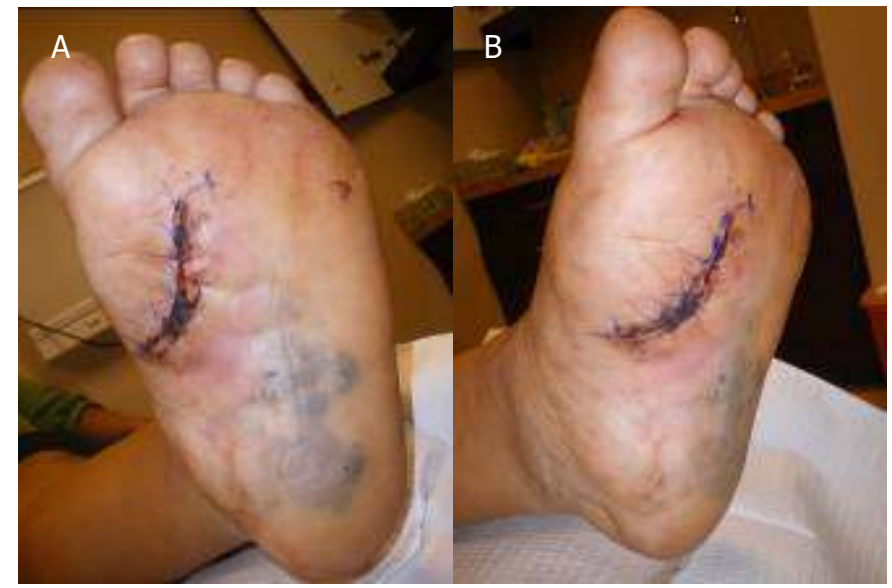

Figure 6A and 6B 2 weeks following the Incision and drainage and foot appears to be healing. (A and $B$ )

The tumors most commonly appear as ballotable masses the overlying skin is usually normal, but may have a bluish hue. Less common dermal involvement manifests as puckering or deep cutaneous dimpling. Lymphangiomas in the subcutis or submucosa manifest as tiny vesicles. Intravascular bleeding is evident by tiny, dark red domed shaped nodules. ${ }^{5}$

Radiologic documentation is best performed by MRI. Ultrasound is a useful auxiliary agent to confirm the presence of macrocystic lymphangiomas. Lymphangiomas, like hemangiomas and most vascular malformations, demonstrate hyperintense signal intensity in T2 weighted and turbo STIR images. Lymphangiomas demonstrate rim enhancement after contrast application. Microcystic lesions have an intermediate signal on T1 sequences and an intermediate to high signal on T2 sequences. Macrocystic lesions show low intensity in T1 and high intensity in T2. Conventional contrast lymphangiography is rarely performed. ${ }^{1,4,7}$

Histologically, lymphangiomas consist of ecstatic lymphatic channels, occasionally containing a mild lymphocytic infiltrate. Classification by histologic appearance includes three subtyes: capillary, cavernous and cystic. The capillary form is composed of small thinned walled lymphatic vessels. Cavernous consists of large lymphatic channels with adventitial coats.
The third and most common type, cystic lymphangioma is composed of macroscopic lymphatic spaces. These lesions are often multiseptated, which is suggestive of infiltration across the tissue planes. ${ }^{1,3}$

Lymphangioma rarely affects the foot and ankle region. Wu in 1996 described a case of a 12 year-old female with a lymphangioma of the anterior aspect of her ankle. He promotes the use of Lymphangiography for diagnosis and recommends for complete surgical excision for treatment. They usually appear in the cervicofacial area up to $75 \%$ of the time. ${ }^{3}$ In 2004, Ly, et al., described another case of lymphangioma in a 2 year-old girl's foot. They recommend Doppler sonography of the mass which reveals no remarkable blood flow. They further describe how to differentiate a lymphangioma from a hemangioma. On MRI, lymphangiomas are characterized by the absence of feeding vessels and lack of intense contrast enhancement. Because of the infiltrating nature of the lesion, complete excision rates are only $18-50 \% .{ }^{1}$ It appears that there have not been a reported percentage of lymphangioma occurrences of the foot and ankle region. In 2009, Itakura, et al., reported 114 cases of lymphangiomas, which of only 2 occurred in the foot. According to their findings the incidence of lymphangioma of the foot is $1.75 \%{ }^{8}$

A purely osseous lymphangioma is a very rare lesion. Bickel and Borders first described lymphangioma of bone in 1947. They described a case of a 5 year girl with an intraosseous lymphangioma of her Ilium. They speculated that as to whether this tumor could have risen in the soft tissue surrounding the bone and caused secondary erosion of the ilium.' In 1977, Jumbelic described the $5^{\text {th }}$ reported case in bone, which was located in the medullary cavity of a long bone, the humerus of a 3 year-old girl. ${ }^{10}$ In 1968, Rosenquist and Wolfe reported one of the first rare cases of an intraosseous lymphangioma of a lower extremity bone. They described a 3 year-old male with lymphangioma of his femur, tibia and fibula. 
Although lymphangiomas are mainly a soft tissue pathology they can occur within bone, but remain extremely rare in the lower extremities. ${ }^{11}$

The two main complications of that can arise from lymphangiomas are intralesional bleeding and infection. Lymphangiomas often swell in the event of a viral or bacterial infection. Most often this is a harmless event likely related to change in flow or alterations. Bacterial cellulitis, however, is more dangerous and requires prolonged intravenous antibiotics. The two main strategies used to treat lymphatic anomalies are schlerotherapy and surgical resection. Schlerotherapy works through obliteration of the lymphatic lumen. Macrocystic lymphangiomas is more likely than microcystic tissue to shrink after an injection of sclerosant. Ethanol is considered to be the most effective sclerosing agent for low-flow malformations, success rates have been reported between $20 \%$ to $65 \%$. Intralesional bleomycin has a reported success of approximately $80 \%$ with lymphangiomas of the head and neck. OK-432, a lyophilized mixture of attenuated group A Streptococcus pyogenes of human origin, has also been reported to have dramatic results reported. Resection is the only way to potentially eliminate the lymphangioma. Complete resection is the operative goal. Resection should be staged and the surgeon should focus on a defined anatomic region. Postoperative wound complications are common, including drainage, seroma and infection. A compressive dressing should be applied immediately post operatively to prevent complications. Even with an intensive approach to resection, the recurrence rate is reported to be $40 \%$ after an incomplete excision and $17 \%$ after a macroscopically complete excision. Angiogenesis inhibitors are at the forefront of medical research for the treatment of venous malformations and neoplasms. Blocking new blood vessel formation may limit the ability of the malformation or neoplasm to fully develop. Approximately 20 angiogenesis inhibitors are being tested in human trials. Research for the development of new therapeutic strategies for vascular tumors by inhibiting angiogenesis is underway. ${ }^{4,5,6}$

(C) The Foot and Ankle Online Journal, 2011
In conclusion, lymphangiomas are rare in the foot and ankle region. A thorough history and physical are necessary to help distinguish from other benign soft tissue tumors. ${ }^{5}$ Imaging studies such as radiographs and MRI are highly recommended to aid in the diagnosis of these tumors. Surgical resection of the mass is recommended for the treatment with the goal of complete excision. The specimen should be sent to pathology for confirmation of the diagnosis. Surgical resection was recommended for this patient. Overall, the patient had minor post operative complications with no sequela. Our recommendation on the day of surgical intervention is to administer a proximal regional block in the popliteal fossa for a longer duration of pain relief post operatively and to admit the patient for observation and pain management. Infection is a common complication for this procedure and the patient should receive pre operative antibiotics and possibly continue them for one week post resection to avoid this complication. The patient was followed for approximately 6 weeks post resection of the tumor and was doing satisfactory, no pain was present upon stance or ambulation. Unfortunately the patient was lost to follow-up due to her relocation to another country.

\section{References}

1. Ly Q, Gilbert B, Davis S, Beall D, Richardson R.

Lymphangioma of the foot. Am J Roent 2005 184:205-206. 2. Skandalakis J, Skandalakis L, Skandalakis P. Anatomy of the lymphatics. Surg Oncol Clin N Am. 2007 16:1-16

3. Wu K. Lymphangioma of the ankle region. J Foot Ankle Surg 1996 35(3):263-65

4. Christison-Lagay E, Fishman S. Vascular anomalies. Surg Clin N Am 2006 86:393-425.

5. Marler J, Mulliken J. Current management of hemangiomas and vascular malformations. Clin Plastic Surg 2005 32:99-116. 6. Dohil M, Baugh W, Eichenfield L. Vascular and pigmented birthmarks. Ped Clin N Am 2000 47(4):783-812.

7. Damron T, Beauchamp C, Rougraff B, Ward W. Soft-tissue lumps and bumps. JBJS 2003 85A:1142-55.

8. Itakura E, Yamamoto H, Oda Y, Furue M, Tsuneyoshi M. VEGF-C and VEGFR-3 in a series of lymphangiomas: is superficial lymphangioma a true lymphangioma? Virchows Arch 2009 454(3):317-259.

9. Bickel W, Broders A. Primary lymphangioma of the ilium: Report of a case. JBJS1947 29A:517-22.

10. Jumbelic M, Feuerstein IM, Dorfman HD. Solitary intraosseous lymphangioma: A case report. JBJS 1984 66A:1479481

11. Rosenquist C, Wolfe D. Lymphangioma of bone. JBJS1968 50A:158-6. 